\title{
High efficiency coronary MRA with non-rigid cardiac motion correction: beyond the quiescent period
}

\author{
Jianing Pang ${ }^{1 *}$, Yuhua Chen ${ }^{1,3}$, Debiao Li ${ }^{1,2}$ \\ From 19th Annual SCMR Scientific Sessions \\ Los Angeles, CA, USA. 27-30 January 2016
}

\begin{abstract}
Background
Coronary arteries remain challenging structures to image using MRI due to the small size, tortuous course, and continual motion. Current high-resolution whole-heart techniques use prospective ECG-triggering and navigator gating to suppress cardiac and respiratory motion artifacts, respectively. However, these motion suppression strategies only accept data acquired within a narrow window in the cardiac and respiratory cycles, resulting in low scanning efficiency and prolonged scan time. Recent works use respiratory motion correction to achieve $100 \%$ respiratory gating efficiency [1-3]. In this work, we extend this concept and develop a non-rigid cardiac motion correction method to extend the cardiac acceptance window beyond the quiescent period.
\end{abstract}

\section{Methods}

MR data was collected using a contrast-enhanced spoiled gradient echo sequence with $3 \mathrm{D}$ radial trajectory and retrospective cardiac and respiratory self-gating, from which 16 cardiac phases were reconstructed with affine respiratory motion correction [4]. Next, all diastolic phases are registered to mid-diastole using a symmetric diffeomorphic model [5]. Then, motion-corrected reconstruction is accomplished by inverting the encoding operator that includes sensitivity encoding and warping the target image to different cardiac phases [6]. Healthy subjects $(\mathrm{N}=7)$ were scanned using a clinical 3T scanner (Siemens Verio). Three images are reconstructed from each dataset: mid-diastole quiescent window, extended window without motion correction, and extended window with motion correction. The scan efficiency, coronary sharpness, and apparent signal-to-noise ratio (aSNR) are compared using paired Student's t-test with a significance level of 0.05 .

\section{Results}

Shown in Fig. 1, the proposed method offers high scanning efficiency, improves aSNR and maintains coronary sharpness over the mid-diastole reconstruction. Fig. 2 shows an example case: a typical mid-diastole window (128 ms) shows little motion blurring but considerable undersampling artifacts; a wide window covering the entire diastole (510 ms) increases aSNR but shows blurring due to cardiac motion; and the motion blurring is effectively removed with cardiac motion correction.

\section{Conclusions}

In this work, we developed a cardiac motion correction framework for high-efficiency coronary MRA, allowing the cardiac acceptance window to be significantly widened while minimizing the artifacts from cardiac motion. The higher scan efficiency can be used to improve the image quality, as shown by our preliminary in vivo studies, or potentially reduce the scan time. Future developments will be focused on optimizing the registration parameters, and further validations on both healthy and patient subjects.

\section{Authors' details}

'Biomedical Imaging Research Institute, Cedars-Sinai Medical Center, Los Angeles, CA, USA. ${ }^{2}$ Bioengineering, University of California, Los Angeles, CA, USA. ${ }^{3}$ Computer and Information Science, University of Pennsylvania, Philadelphia, PA, USA. 


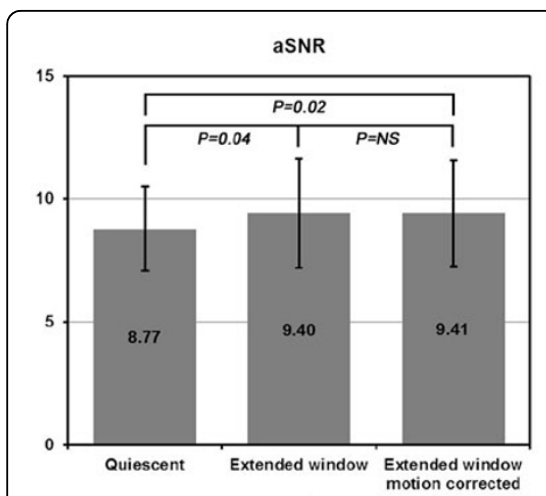

(a)

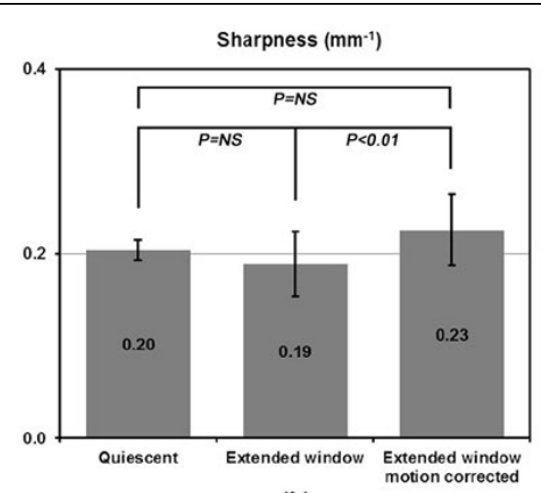

(b)

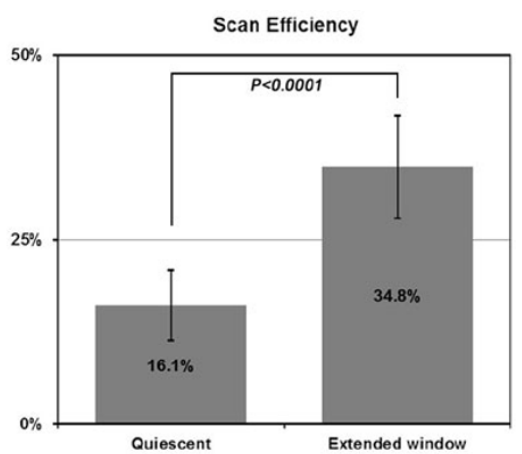

(c)

Figure 1 Quantitative comparisons: (a) accepting more data with an extended window improves aSNR significantly over the middiastole window; (b) the residual cardiac motion within the wide acceptance window decreases the coronary sharpness, which is recovered by performing cardiac motion correction; (c) the proposed method significantly increases the imaging efficiency.

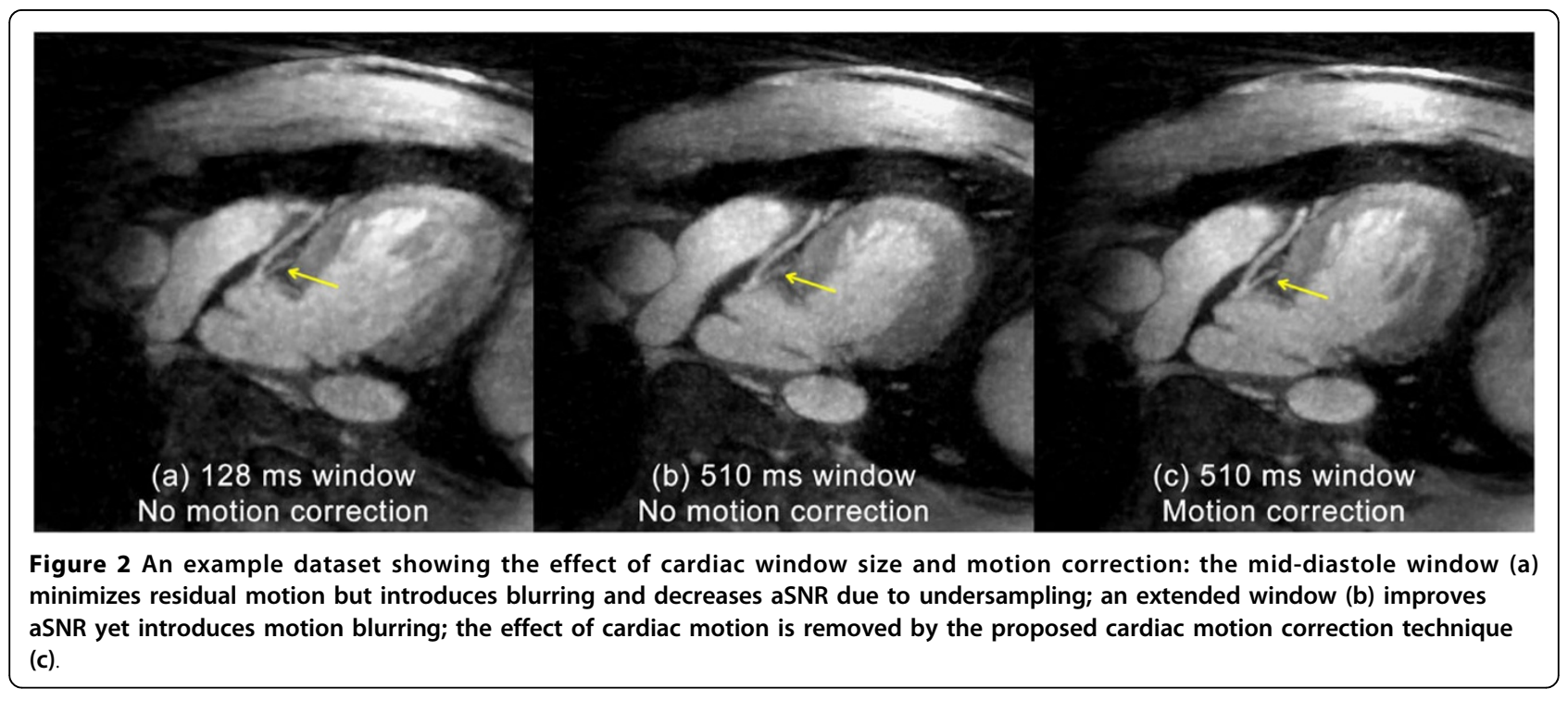

Published: 27 January 2016

\author{
References \\ 1. Piccini : MRM 2012. \\ 2. Pang: MRM 2014. \\ 3. Henningsson : MRM 2014. \\ 4. Pang: MRM 2014. \\ 5. Avants: Med Image Anal 2008. \\ 6. Schmidt : MRM 2011
}

doi:10.1186/1532-429X-18-S1-0107

Cite this article as: Pang et al:: High efficiency coronary MRA with nonrigid cardiac motion correction: beyond the quiescent period. Journal of Cardiovascular Magnetic Resonance 2016 18(Suppl 1):0107.

\section{Submit your next manuscript to BioMed Central} and take full advantage of:

- Convenient online submission

- Thorough peer review

- No space constraints or color figure charges

- Immediate publication on acceptance

- Inclusion in PubMed, CAS, Scopus and Google Scholar

- Research which is freely available for redistribution 